\title{
Supervivencia de pacientes con cáncer de próstata en un hospital de Bogotá, Colombia 2008-2014
}

\section{Survival of patients with prostate cancer in a hospital Bogotá, Colombia 2008-2014}

\author{
Nelson Rolando Campos-Guzmán (iD) ${ }^{1}$ \\ 1. Universidad de Ciencias Aplicadas y Ambientales (UDCA). Bogotá, Colombia. Correo: ncampos@udca.edu.co - https://orcid.org/0000-0002- \\ 1102-7116
}

Tipología: Artículo de investigación científica y tecnológica

Para citar este artículo: Campos-Guzmán NR. Supervivencia de pacientes con cáncer de próstata en un hospital de Bogotá, Colombia 2008-2014. Duazary. 2021 julio; 18(3): 259-268. Doi: https://doi.org/10.21676/2389783X.4238

Palabras clave: neoplasias de la próstata; análisis de supervivencia; pronóstico.

\section{RESUMEN}

El cáncer de próstata es actualmente una de las principales patologías que afectan a los hombres. El objetivo del presente artículo fue estimar la supervivencia de los pacientes con cáncer de próstata de riesgo intermedio y alto pertenecientes al régimen especial de salud (militares). Se realizó un estudio de cohorte que estima la función de supervivencia con el método de Kaplan-Meier y el Hazard ratio (HR) en función de variables explicativas mediante un modelo de regresión de Cox. Los pacientes incluidos fueron diagnosticados por urología y/o confirmados con apoyos diagnósticos. No se incluyeron pacientes tratados para cáncer de próstata en otra institución o por tumores primarios diferentes a próstata y quienes consultaron para tomar una segunda opinión médica. Los pacientes de riesgo intermedio sobreviven en un $90 \%$ tanto a cinco como a siete años, y los de riesgo alto sobrevivieron en $35 \%$ y $30 \%$ a cinco y siete años, respectivamente, con una mediana de 28 meses. Este estudio permitió concluir que la supervivencia de los pacientes clasificados como riesgo intermedio es similar a la nacional e internacional; sin embargo, en los pacientes de alto riesgo la supervivencia es inferior respecto a otros países.

\section{ABSTRACT}

Keywords: Prostatic Neoplasms; Survival Analysis; Prognosis.
Prostate cancer is currently one of the main pathologies that affects men. The objective of this article was to estimate the survival of patients with intermediate and high risk prostate cancer belonging to the special health regimen. A cohort study was conducted that estimates the survival function with the Kaplan-Meier method, the Hazard ratio (HR) as a function of explanatory variables using a Cox regression model. The patients included were diagnosed by urology and / or confirmed with diagnostic support, patients treated for prostate cancer in another institution, primary tumors other than the prostate, and those who consulted for a second medical opinion were not included. Intermediate-risk patients survive $90 \%$ to both 5 and 7 years, and high-risk patients survive 35\% and 30\% to 5 and 7 years, respectively, with a median of 28 months. This study allowed us to conclude that survival in patients classified as intermediate risk is similar to national and international ones, however, in high-risk patients, survival is lower than in other countries. 


\section{INTRODUCCIÓN}

El cáncer de próstata ( $\mathrm{CaP}$ ) representa una de las neoplasias más frecuentes en los hombres del mundo occidental. Además, es la primera causa de atención en la consulta de uro-oncología ${ }^{1}$. Este es el segundo cáncer más frecuente en los hombres, después del de pulmón, y la quinta causa principal de muerte por cáncer. El CaP tiene una esperanza de vida larga, aunque su supervivencia varía de forma importante en función de la edad, el estadio y la puntuación en la escala de Gleason, factores pronósticos descritos ampliamente en la literatura ${ }^{2}$.

Según el Instituto Nacional de Cáncer de Estados Unidos, el CaP predomina en hombres de edad avanzada, su tasa de crecimiento tumoral oscila entre muy lento a moderadamente rápido, y la supervivencia en la mayoría de pacientes es prolongada. La tasa de supervivencia relativa a cinco años del diagnóstico con enfermedad local o regional fue de $100 \%$, y la proporción de enfermedad a distancia fue de $28,7 \%$. Se observó una tasa de supervivencia del $99 \%$ para todos los estadios combinados ${ }^{3}$.

En Latinoamérica, la incidencia de CaP varía en cada país. Sin embargo, no es posible determinar la real incidencia puesto que no todos los países cuentan con un registro poblacional de cáncer. Acorde a la información reportada, la morbilidad y mortalidad atribuida a este cáncer va en aumento: la incidencia en San Pablo (Brasil) fue de 65,1 por 100.000 hombres; en Quito (Ecuador), de 31,3 por 100.000, y en Colombia, de 47,7 por 100.000 hombres $^{4}$.

En Colombia la tendencia de mortalidad general por cáncer va en aumento, tanto en niños como en adultos, aunque es importante resaltar que el cáncer de estómago y el de cuello uterino, que se encuentran entre los de mayor incidencia y mortalidad, han registrado una tendencia al descenso. Entre los hombres, las principales localizaciones de cáncer fueron, en orden decreciente: próstata, estómago, pulmón, colón, recto y linfomas no-Hodgking. El mayor número de muertes se presentan en cáncer de estómago con $14,9 \%$, pulmón con $12,3 \%$, próstata con $7,3 \%$ y mama con $6,2 \%{ }^{5}$.
La supervivencia global de los pacientes con cáncer de próstata aporta para la toma de decisiones terapéuticas, orienta para definir protocolos de seguimiento e, igualmente, apoya la sustentación de políticas en salud. No obstante, en Colombia es limitada la información en relación con la supervivencia de $\mathrm{CaP}$ en pacientes afiliados al régimen especial de cobertura a militares, el cual se rige por normas legales concebidas antes de la entrada en vigencia de la Ley 100 de 1993, al igual que con respecto al peso de los factores pronósticos. Esta situación ha motivado el desarrollo del presente estudio, con el objetivo de estimar la supervivencia de los pacientes con CaP de riesgo intermedio y alto pertenecientes al régimen especial diagnosticados en el periodo 2008-2014, en un hospital de la ciudad de Bogotá D. C., Colombia.

\section{MATERIALES Y MÉTODOS}

\section{Tipo de investigación}

Cohorte histórica a través de la revisión de registros en las historias clínicas.

\section{Población y muestra}

Se consultaron un total de 180 historias clínicas del archivo de consulta externa del servicio de oncología filtradas por el diagnóstico CaP. De ellas, 123 cumplieron los criterios de inclusión, esto es: diagnóstico de $\mathrm{CaP}$ por médico especialista (urología) y/o confirmado con apoyos diagnósticos en consultas registradas en la historia clínica de primera vez a partir del 1 de enero del año 2008 hasta el 31 de diciembre del año 2014. Se excluyeron pacientes intervenidos por cáncer de próstata previamente en otra institución, pacientes con tumores primarios diferentes a próstata $y$ registros inconsistentes o incompletos en la historia clínica. Como variable desenlace se tomó fallecimiento del paciente.

\section{Procedimiento}

La información obtenida se organizó en dos grupos acordes a la categoría de riesgo D'Amico (riesgo intermedio: tumor que ha invadido el lóbulo 
contrario de la próstata y tiene puntuación de Gleason igual a 7 o una concentración de PSA de entre 11 y $19 \mathrm{ng} / \mathrm{ml}$; categoría de riesgo alto: tumor que ha invadido las estructuras adyacentes a la próstata, presenta una puntuación Gleason superior a 7 o una concentración de PSA mayor de $20 \mathrm{ng} / \mathrm{ml})^{6}$. Para el análisis de supervivencia se tomaron la fecha inicial (confirmación del diagnóstico por médico especialista) y la fecha de último contacto acorde a la de defunción registrada en la historia clínica. Se tuvo en cuenta la discriminacion por factores pronósticos descritos en la literatura como: concentración del antígeno prostático, puntuación Gleason (grado de diferenciacion celular) y edad.

\section{Análisis de datos}

Los datos se procesaron en el programa Stata version 17.0. Se realizó un análisis descriptivo bivariado a partir de la variable dependiente (fallecimiento). Para el análisis de supervivencia se utilizó el método de Kaplan-Meier ${ }^{7}$ y se estimó el Hazard ratio (HR) crudo a cada una de las variables pronóstico (edad, puntuación Gleason, PSA, metástasis). Las curvas de supervivencia entre los grupos de mediano y alto riesgo se compararon con la prueba de logaritmo de rango, se construyó el modelo de regresión de Cox, y posteriormente se validó con la prueba de hipótesis de riesgos proporcionales.

\section{Declaración sobre aspectos éticos}

De acuerdo con los principios establecidos en la Declaración de Helsinki y en la Resolución 8430 de octubre 4 de $1993^{8}$, esta investigación se considera sin riesgo y cumple con los aspectos mencionados en la normativa vigente. El protocolo de investigación fue sometido y aprobado por el comité de ética de la institución hospitalaria.

\section{RESULTADOS}

Los pacientes del estudio provenían de 20 departamentos de Colombia: predominantemente, de la capital del país, Bogotá D. C., con un 24\%, seguida de los departamentos de Boyacá y Cundinamarca, cada uno de ellos con un $15 \%$, y el $46 \%$ restante correspondían a otros departamentos.
Para el análisis descriptivo se tuvieron en cuenta variables sociodemográficas, factores pronósticos y características clínicas y patológicas de los pacientes en estudio (tabla 1).

De los pacientes estudiados, el $32 \%$ se clasificaron en riesgo intermedio, y $68 \%$, en alto riesgo. Sin embargo, el porcentaje de pacientes con cáncer de próstata consultantes ha venido presentado una disminución importante a partir del año 2008. Quienes requieren la atención por hematooncología se encuentran en estadios avanzados (figura 1).

En el análisis bivariado se halló que un $54 \%$ de los pacientes que fallecieron eran mayores de 75 años; específicamente, pertenecían al grupo etario de 7180 años $(p=0,004)$. Por otra parte, en cuanto a las variables antecedentes de cáncer de próstata, raza y nivel de escolaridad, no se encontró significancia estadística. Los pacientes negaron antecedentes familiares de cáncer de próstata en un $93 \%$ $(p=0,296)$; un $52 \%$ eran de raza mestiza, y $48 \%$, de raza blanca ( $p=0,609)$, y un $44 \%$ de los pacientes que fallecieron tenían un nivel de escolaridad primaria $(p=0,149)$.

EI PSA de los pacientes que fallecieron en el momento de consulta a urología era mayor e igual a $20 \mathrm{ng} / \mathrm{ml}$ en un $83 \%(p<0,001)$; para los que fallecieron en el momento de consulta a hematooncología, se encontraba mayor e igual a $20 \mathrm{ng} / \mathrm{ml}$ en un $64 \%(p<0,001)$, y los de fallecimiento posterior al tratamiento reflejaron un PSA mayor e igual a 20 $\mathrm{ng} / \mathrm{ml}(\mathrm{p}<0,001)$ en un $71 \%$ de los casos. 
Tabla 1. Características clínicas y patológicas.

\begin{tabular}{|c|c|c|c|}
\hline Variable & Número (\%) & Variable & Número (\%) \\
\hline Edad & $73 \mathrm{DS}=8,9$ años & Metástasis & \\
\hline Edad agrupada & \multirow{3}{*}{$\begin{array}{l}11(9) \\
37(30) \\
54(44) \\
21(17)\end{array}$} & $\begin{array}{c}\text { Mixta } \\
\text { Óseas } \\
\text { Visceral } \\
\text { No metástasis }\end{array}$ & $\begin{array}{l}10(8) \\
57(47) \\
5(4) \\
51(41)\end{array}$ \\
\hline $\begin{array}{l}\text { Menores e iguales a } 60 \text { años } \\
61-70 \text { años }\end{array}$ & & Antígeno prostático PSA & \multirow[b]{2}{*}{$\begin{array}{l}61(49,6) \\
62(50,4)\end{array}$} \\
\hline $\begin{array}{l}\text { 71-80 años } \\
\text { Mayores a } 81 \text { años }\end{array}$ & & $\begin{array}{c}\text { PSA mayor a } 20 \mathrm{ng} / \mathrm{ml} \\
\text { PSA menor o igual a } 20 \mathrm{ng} / \mathrm{ml}\end{array}$ & \\
\hline Categoría de riesgo & \multirow{2}{*}{$\begin{array}{l}39(32) \\
84(68)\end{array}$} & $\begin{array}{c}\text { Antígeno prostático posterior } \\
\text { al tratamiento Hemato- } \\
\text { oncología (solo pacientes de } \\
\text { riesgo alto) }\end{array}$ & \\
\hline $\begin{array}{l}\text { Riesgo intermedio } \\
\text { Riesgo alto }\end{array}$ & & $\begin{array}{c}\text { PSA mayor a } 20 \mathrm{ng} / \mathrm{ml} \\
\text { PSA menor o igual a } 20 \\
\mathrm{ng} / \mathrm{ml} \\
\text { No tratamiento por } \\
\text { hemato-oncología }\end{array}$ & $\begin{array}{l}15(12) \\
26(21) \\
82(67)\end{array}$ \\
\hline \multicolumn{4}{|l|}{ Puntuación escala Gleason } \\
\hline $\begin{array}{c}\text { Inferior a } 7 \\
\text { Mayor igual a } 7\end{array}$ & $\begin{array}{l}42(34) \\
81(66)\end{array}$ & & \\
\hline
\end{tabular}

DS: desviación estándar. PSA: antígeno prostático.

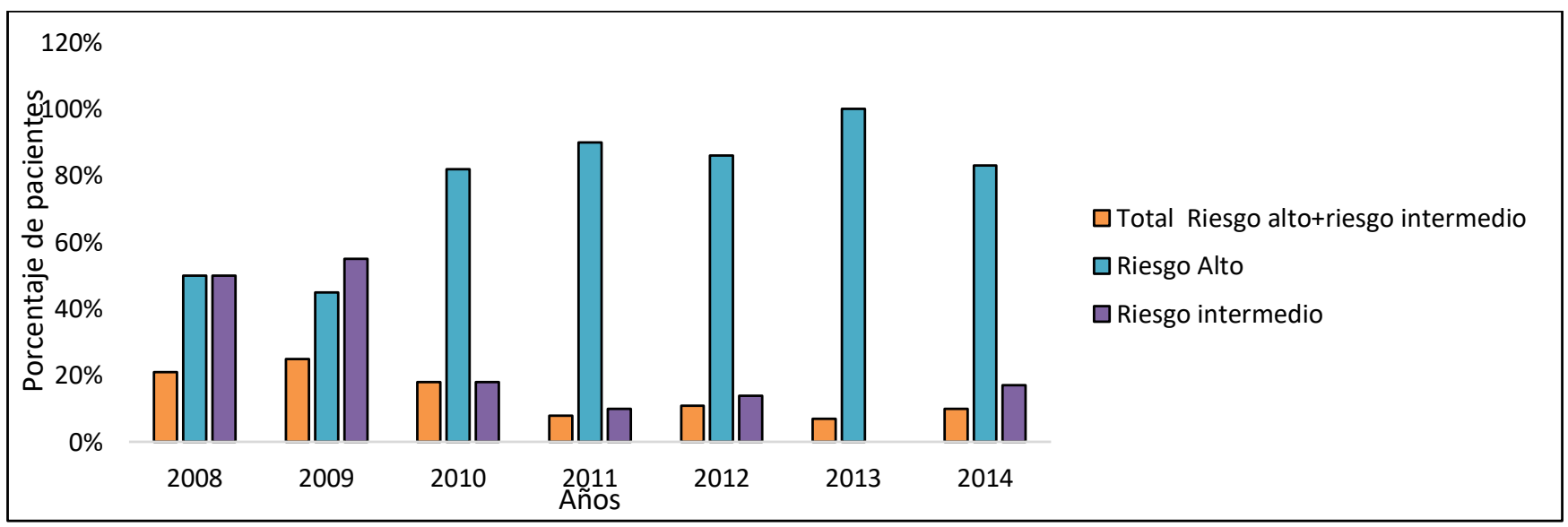

Figura 1. Porcentaje de pacientes con diagnóstico de cáncer de próstata. 
En relación con el grado de diferenciación del tumor y el evento de interés, se obtuvo que un $82 \%$ de los pacientes que fallecieron tenían una puntuación Gleason mayor a $7(p=0,02)$, la extensión tumoral T4 se halló en un $89 \%$, y no se pudieron evaluar ganglios linfáticos regionales en el $93 \%$ de los pacientes $(p=0,003)$. La metástasis a distancia en tejido óseo (M1b) estuvo presente en el $83 \%$ de los pacientes $(p<0,001)$.

Finalmente, de los pacientes que fallecieron, el $94 \%$ estaban clasificados en alto riesgo, y el $6 \%$ restante eran de riesgo intermedio $(p<0,001$.

\section{Análisis de supervivencia}

La sobrevida global de los pacientes con cáncer de próstata a cinco años fue de $57 \%$, y a siete años, del $55 \%$. En los primeros 36 meses, un $40 \%$ de los pacientes fallecieron. La supervivencia de los pacientes de riesgo intermedio fue de un $90 \%$ tanto a cinco como a siete años, mientras que para los pacientes de riesgo alto fue de $35 \%$ y $30 \%$ a cinco y siete años, respectivamente, con una mediana de 28 meses (figura 2).

Por otro lado, la sobrevida de los pacientes con cáncer de próstata que presentan metástasis es de $27 \%$ a cinco años y $20 \%$ a siete años, con una mediana de 23 meses, y los pacientes que no presentan cáncer de próstata metastásico sobreviven en un $90 \%$ a cinco y a siete años.

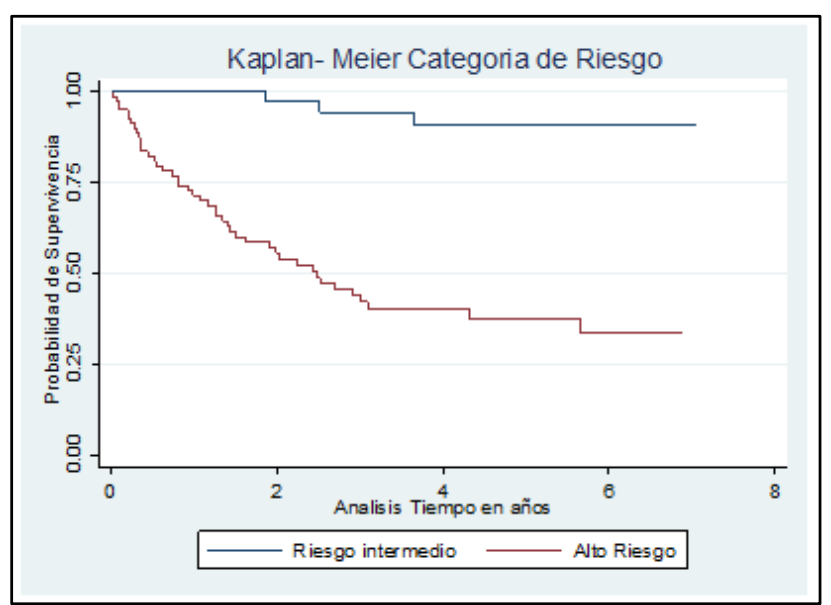

Figura 2. Supervivencia de los pacientes con cáncer de próstata por categoría de riesgo $(p=0,01)$.
Las diferencias en supervivencia se encontraron específicamente en los pacientes clasificados como de alto riesgo. Los siguientes resultados se presentarán en función de este grupo de pacientes.

\section{Sobrevida en cuanto a factores pronósticos en pacientes de alto riesgo}

En relación con el puntaje de Gleason para los pacientes de alto riesgo, no se encuentran diferencias estadísticas en la supervivencia log Rank $(p=0,68)$. Aquellos pacientes con una puntuación Gleason mayor e igual a 7 tienen una tasa de sobrevida a cinco años del $24 \%$ y a siete años del $10 \%$, con una mediana de 18 meses, mientras que los pacientes que tienen una puntuación Gleason menor a 7 sobreviven a cinco años un $30 \%$ y a siete años un $28 \%$, con una mediana de 27 meses.

En cuanto a la edad, los pacientes menores e iguales a 60 años presentan una sobrevida del $100 \%$ a siete años; los de 61 a 70 años muestran una sobrevida del $68 \%$ a siete años; los de 71 a 80 años reflejan una sobrevida del $45 \%$ a siete años, con una mediana de 47 meses, y pacientes mayores a 81 años tienen una sobrevida del $32 \% \log \operatorname{Rank}(p=0,021)$ (figura 3 ).

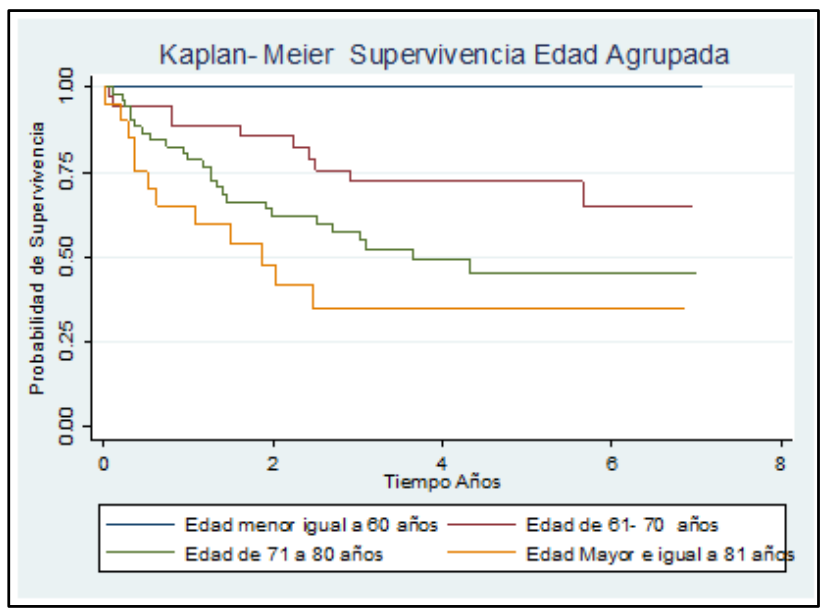

Figura 3. Supervivencia de los pacientes con cáncer de próstata de mediano y alto riesgo por grupos etarios. 
Tabla 2. Hazard ratio (HR) de los pacientes de alto riesgo.

\begin{tabular}{|c|c|c|c|}
\hline Variable & $\begin{array}{l}\text { Hazard ratio } \\
\text { (HR) }\end{array}$ & p & IC95\% \\
\hline $\begin{array}{l}\text { Antígeno prostático en el momento de diagnóstico de cáncer de } \\
\text { próstata mayor a } 20 \mathrm{ng} / \mathrm{ml}\end{array}$ & 3,7 & 0,008 & $1,41-9,69$ \\
\hline $\begin{array}{l}\text { Antígeno prostático al inicio del manejo clínico por servicio de hemato- } \\
\text { oncología mayor a } 20 \mathrm{ng} / \mathrm{ml}\end{array}$ & 3,49 & 0,050 & $0,99-6,23$ \\
\hline $\begin{array}{l}\text { Antígeno prostático en consulta posterior a tratamiento de hemato- } \\
\text { oncología }\end{array}$ & 8,5 & 0,007 & $1,80-39,9$ \\
\hline Edad mayor a 72 años & 14 & 0,009 & 1,93-102 \\
\hline Presencia de metástasis & 21 & 0,001 & $6,51-68$ \\
\hline Puntuación Gleason mayor e igual a 7 & 2,92 & 0,03 & $1,1-7,74$ \\
\hline
\end{tabular}

Tabla 3. Modelo de regresión de Cox para pacientes con cáncer de próstata de alto riesgo.

\begin{tabular}{|c|c|c|}
\hline Evento muerte & HR & Valor de $p$ \\
\hline Cáncer de próstata con metástasis & 13,1 & 0,02 \\
\hline $\begin{array}{l}\text { Antígeno prostático al inicio del manejo clínico por servicio de hemato-oncología mayor } \\
\qquad \text { a } 20 \mathrm{ng} / \mathrm{ml}\end{array}$ & 7 & 0,005 \\
\hline
\end{tabular}

HR: Hazard ratio.

En cuanto a la cinemática del PSA en diferentes momentos, no se encontraron diferencias en el momento de la consulta con urología por primera vez log Rank $(p=0,22)$. Sin embargo, se halló diferencia en la supervivencia en cuanto al PSA tomado en la consulta por hemato-oncología. Entre quienes tuvieron un PSA mayor e igual a $20 \mathrm{ng} / \mathrm{ml}$, la sobrevida a cinco años fue del $24 \%$, con una mediana de 20 meses, mientras que para los pacientes que reportaron un PSA menor a $20 \mathrm{ng} / \mathrm{ml}$ la sobrevida a cinco años fue del $30 \%$, con una mediana de 50 meses log Rank ( $p=0,044)$.

Acorde a lo propuesto en la metodología, no solo se estimó la relación de tasa de supervivencia, sino su relación entre las variables pronósticas, ajustándolas mediante un modelo de regresión de $\operatorname{Cox}^{9}$ (Tabla 2).

Como se propuso en la metodología, para desarrollar el modelo se incluyeron cada una de las variables con significancia estadística y se intentó preservar las variables clínicas descritas en la literatura. De esta forma quedó un modelo con dos variables: cáncer de próstata con metástasis y antígeno prostático al inicio del manejo clínico por hemato-oncología mayor a $20 \mathrm{ng} / \mathrm{ml}$ (tabla 3).

Se verificó la proporcionalidad de los riesgos durante el tiempo para este modelo aceptando la hipótesis nula; por tanto, este modelo es válido $(p=0,99)$. Esta prueba estuvo basada en los residuos de Schoenfeld, los cuales se calcularon para cada predictor y para cada paciente.

\section{DISCUSIÓN}

Este estudio proporciona estimaciones de la supervivencia global de los pacientes con $\mathrm{CaP}$ del $57 \%$ a cinco años y del $55 \%$ a siete años. Al analizar por categoría de riesgo, se observa variabilidad en los pacientes de alto riesgo con una sobrevida del $35 \%$ a cinco años y del $30 \%$ a siete años, versus los pacientes de riesgo intermedio, en los que se estimó una sobrevida del $90 \%$ a los cinco y siete años.

El estudio Concord-2 refiere aumento de la supervivencia entre $10-20 \%$ a cinco años en 22 países de América del Sur, Asia y Europa. Sin embargo, existe variabilidad en todo el mundo: 
desde menos del $60 \%$ en Bulgaria a $95 \%$ en Tailandia ${ }^{10}$. Específicamente en Colombia, para el año 1999 la supervivencia era del 68\%, y para el año 2009 ya fue del $78 \%$. Al analizar la supervivencia en el país por regiones, se observa que en el centro la sobrevida global a cinco y diez años en etapa localizada fue del $92 \%$ y del $79 \%$; en enfermedad metastásica, de $52 \%$ y $30 \%$, y con enfermedad regional la sobrevida fue de $100 \%$ tanto a cinco como a diez años respectivamente ${ }^{11}$. Entretanto, en ciudades específicas como Cali, la supervivencia global de pacientes con CaP entre los años 1962 al 2011 fue de 69,8\% a cinco años (IC95\% 67,5-72) ${ }^{12}$.

Así pues, comparando los resultados del estudio Concord-2 y los estudios locales con la presente investigación, se observa que la supervivencia de los pacientes de riesgo intermedio se comporta de manera similar. No obstante, cuando se analiza la supervivencia en el grupo de pacientes de alto riesgo del régimen especial, esta es inferior. La supervivencia de los pacientes del régimen especial de este estudio se asocia a factores descritos en la literatura como la edad, el estadio clínico (TNM), el tratamiento primario con radioterapia, los niveles de PSA, la raza y el puntaje de Gleason (mayor o igual a 7) ${ }^{13-17}$.

En relación con la edad, en el grupo de riesgo intermedio el rango fue de 46 a 83 años, y en el grupo de alto riesgo, entre 55 y 94 años. Para este estudio el promedio de edad fue de 73 años, lo que coincide con la afirmación de DeVita et a ${ }^{18}$ : "no hay otro tumor en adultos que esté más relacionado con la mayor edad que el cáncer de próstata". Sin embargo, en los pacientes de alto riesgo la media de edad fue de 76 años, datos estadísticos similares a los reportados por el National Institute of Health de Estados Unidos ${ }^{4}$, el cual informa de un promedio de 72 años.

En cuanto a los antecedentes familiares de cáncer de próstata como factor pronóstico, se obtiene que la prevalencia para este estudio es del $7 \%$, similar a la hallada en el estudio de Rodríguez et al. ${ }^{19}$, del $9 \%$. La literatura afirma que el riesgo se duplica en los pacientes que tienen antecedentes de $\mathrm{CaP}^{20}$; por lo tanto, se requiere intensificar los programas de atención primaria para el diagnóstico oportuno entre los familiares de estos pacientes en estadios tempranos, que es cuando se presenta mayor supervivencia.

Con respecto al factor pronóstico puntuación Gleason, los resultados fueron consistentes con otros estudios. En el $82 \%$ de los pacientes que fallecieron en el presente estudio la puntuación Gleason fue mayor o igual a 7, y su HR crudo fue de 2,92. Por su parte, Segarra et al. ${ }^{21}$ encontraron que a mayor puntuación Gleason, la probabilidad de cáncer de próstata no localizado se incrementa. Este hallazgo es ratificado por Pound et $a l^{22}$, quienes concluyen que la puntuación Gleason mayor o igual a 8 es predictor significativo de recurrencia a distancia. En este estudio los pacientes que tenían puntuación de Gleason mayor 0 igual a 7 desarrollaron metástasis en un $85 \%(p<0,001)$. De esta manera se podría inferir que la puntuación de Gleason cobra un peso importante en los pacientes de riesgo intermedio, pero no en aquellos que presentan metástasis.

Otra de las variables pronóstico es el PSA. En este estudio, el $95 \%$ de los pacientes presentaron un valor mayor al de referencia $(4 \mathrm{ng} / \mathrm{ml})$, y puede establecerse que el PSA mayor a $20 \mathrm{ng} / \mathrm{ml}$ al inicio del tratamiento por hemato-oncología incrementa el riesgo de morir 3,4 veces (IC95\% 1,27-9,46). En relación con los pacientes que debutan con un PSA mayor a $20 \mathrm{ng} / \mathrm{ml}$, la supervivencia a siete años es del $45 \%$, mientras que en aquellos casos en que, posterior al tratamiento, los tumores son resistentes a la hormono-castración la supervivencia es del $8 \%$, con un promedio de supervivencia de 12 meses.

Para este estudio la variable PSA mayor a $20 \mathrm{ng} / \mathrm{ml}$ en el momento de consulta por hemato-oncología hace parte del modelo final con un HR de 7 $(p=0,005)$, en presencia de la variable cáncer de próstata metastásico con HR de $13(p=0,02)$. Si bien es cierto que el PSA no es una prueba para tamización por su baja sensibilidad, sí se convierte en un factor pronóstico en los pacientes con estadios avanzados de la enfermedad, pues este incremento del PSA obedece a una enfermedad resistente a la hormono-castración.

Por último, el PSA y la puntuación de Gleason son los dos factores predictivos más importantes respecto a la aparición de metástasis óseas ${ }^{19}$. 


\section{CONCLUSIÓN}

En este estudio se pudo determinar que la supervivencia de los pacientes que reciben atención en el régimen especial y son clasificados como de riesgo intermedio es similar a la reportada en los estudios nacionales e internacionales. Sin embargo, en los pacientes de alto riesgo de la presente investigación la supervivencia fue inferior con relación a los estudios mencionados. El mayor número de fallecimientos ocurren en los primeros tres años posteriores al manejo por hematooncología.

La cantidad de casos nuevos diagnosticados de CaP en hombres afrodescendientes es casi un $80 \%$ más alta que la cantidad de casos nuevos diagnosticados en hombres caucásicos ${ }^{22,23}$. No obstante, por razones como la ubicación geográfica, el porcentaje de pacientes afrodescendientes fue nulo para este estudio, por lo que el modelo y los resultados no se pueden extrapolar a los pacientes de este grupo poblacional.

Por otra parte, no fue posible conocer el tiempo de doblaje del antígeno prostático PSA doble time puesto que no se registraba la fecha de este en la historia clínica, si bien esta es una variable que puede explicar el tiempo de supervivencia en los pacientes con cáncer de próstata metastásico e, igualmente, las recidivas bioquímicas.

\section{RECOMENDACIONES}

Se recomienda desarrollar estudios periódicos multicéntricos partiendo de los registros poblacionales de cáncer unificados.

\section{DECLARACIÓN SOBRE CONFLICTOS DE INTERESES}

El autor declara que no existe conflicto de intereses.

\section{REFERENCIAS BIBLIOGRÁFICAS}

1. Ribal M, Mellado B. Guías de consenso para el manejo del cáncer de próstata resistente a castración en Catalunya. UOC; 2014. Disponible en: https://books.google.com/books?hl=es \&lr=\&id=0q HNAgAAQBAJ\&oi=fnd\&pg=PT4\&dq=B. $+\mathrm{Gu} \% \mathrm{C} 3 \% \mathrm{AD}$ as+de+consenso+para+el+manejo+del+c\%C3\%A1nc er+de+pr\%C3\%B3stata+resistente+a+castraci\%C3\% B3n+en+Catalunya\&ots $=5$ szJGStLO1\&sig $=5$ WUCJED d2zFMVtzkuCXP3gPLjd4

2. Campá J, Mar B, Extramiana J, Arróspide A, Mar J. Supervivencia del cáncer de próstata avanzado en España según escala de Gleason, edad y estadio. Actas Urológicas Españolas. 2016 Oct; 40(8): 499506.

Doi:

https://doi.org/10.1016/j.acuro.2016.03.0083

3. National Cancer Institute. Prostate Cancer Prevention $\left(\mathrm{PDQ}^{\circledR}\right)-$ Health Professional [Internet] [citado 15 sep 2019]. Disponible en: https://www.cancer.gov/types/prostate/hp/prosta te-prevention-pdq

4. Pow-Sang M, Destefano V, Astigueta J, Castillo O, Gaona J, Santaella $F$, et al. Cáncer de próstata en Latinoamérica. Actas Urol Esp. 2009 Dic; 33(10): 1057-61. Doi: https://doi.org/10.1016/S02104806(09)73181-X

5. Ospina M, Huertas J, Montaño J, Rivillas J. Observatorio Nacional de Cáncer Colombia. Fac. Nac. Salud Pública [revista en la Internet]. 2015 May; 33(2): 262-76. Disponible en: https://revistas.udea.edu.co/index.php/fnsp/article /view/19044

6. Gutiérrez P, González S, Conde V. Influencia del protocolo diagnóstico y la edad en la tasa de incidencia de cáncer de próstata en Castilla y León según el registro nacional de 2010. Actas Urol Esp. 2021; 45(5): 383-90. Doi: https://doi.org/10.1016/j.acuro.2020.11.007

7. Lacny S, Wilson $T$, Clement F, Roberts $D$, Faris $P$, Ghali W, et al. Kaplan-Meier survival analysis overestimates cumulative incidence of healthrelated events in competing risk settings: a metaanalysis. Journal of Clinical Epidemiology. 2018 Oct; 93: 25-35. Doi: https://doi.org/10.1016/j.jclinepi.2017.10.006 
8. Colombia. Ministerio de Salud. Resolución Número 8430 de 1993 [Internet]. Disponible en: https://www.minsalud.gov.co/sites/rid/Lists/Bibliot ecaDigital/RIDE/DE/DIJ/RESOLUCION-8430-DE1993.PDF

9. Montero Granados R. Modelos de regresión lineal múltiple [Trabajo de grado Economía Aplicada]. España: Universidad de Granada. Facultad Ciencias Empresariales; 2016. Disponible en: http://www.ugr.es/ montero/matematicas/regresi on_lineal.pdf

10. Allemani C, Weir H, Carreira H, Harewood R, Spika D, Wang $X$, et al. Global surveillance of cancer survival 1995-2009: Analysis of individual data for 25 676887 patients from 279 population-based registries in 67 countries (Concord-2). Lancet. 2015; 385(9972): 977-1010. Doi: https://doi.org/10.1016/S0140-6736(14)62038-9

11. Villegas C, Chacón J, Sánchez T. Sobrevida en cáncer de próstata de una población del centro de Colombia 1997-2012. Acta Médica Colombiana. 2015; 40(2): 101-8. Doi: https://doi.org/10.36104/amc.2015.447

12. Restrepo J, Bravo L, García-Perdomo H, García L, Collazos P, Carbonell J. Incidencia, mortalidad y supervivencia al cáncer de próstata en Cali, Colombia, 1962-2011. Salud Pública Mex. 2014; 56(5): 440-7. Doi: https://doi.org/10.21149/spm.v56i5.7369

13. Yossepowitch O, Eggener S, Serio A, Carver B, Bianco F, Scardino P, et al. Secondary Therapy, Metastatic Progression, and Cancer-Specific Mortality in Men with Clinically High-Risk Prostate Cancer Treated with Radical Prostatectomy. Eur Urol. 2008; 53(5): 950-9. Doi: https://doi.org/10.1016/j.eururo.2007.10.008

14. Angulo J, Romero I, Díaz M, Enrech S, Díez R, Molina T. Supervivencia del cáncer de próstata resistente a la castración en la práctica clínica y el papel del tratamiento. Rev Colombiana de Cancerología. 2017 Jun; 21(2): 95-103. Doi: http://dx.doi.org/10.1016/j.rccan.2017.03.002
15. Yamamoto S, Kawakami S, Yonese J, Fujii $Y$, Urakami S, Kitsukawa S, et al. Long-term oncological outcome inmen with T3 prostate cancer: Radical prostatectomy versus external-beam radiation therapy at a single institution. Int J Clin Oncol. 2014; 19: 1085-91. Doi: https://doi.org/10.1007/s10147013-0654-2

16. Abdollah F, Schmitges J, Sun M, Jeldres C, Tian Z, Briganti $A$, et al. Comparison of mortality outcomes after radical prostatectomy versus radiotherapy in patients with localized prostate cancer: A population-based analysis. Int J Urol. 2012; 19(9): 836-44. Doi: https://doi.org/10.1111/j.14422042.2012.03052.x

17. Neeple K, Stephenson A, Kallogjeri D, Michalski J, Grubb I, Strope S, et al. Mortality After Prostate Cancer Treatment with Radical Prostatectomy, External-Beam Radiation Therapy, or Brachytherapy in Men Without Comorbidity. European Urology. 2013; 64(3): 372-8. Doi: https://doi.org/10.1016/j.eururo.2013.03.005

18. DeVita V, Hellman S, Rosenberg S. Cancer Principles \& Practice of Oncology. 6. ${ }^{\text {a }}$ edición. [Internet]. Philadelphia: Williams \& Wilkins Publishers; 2011. Disponible en: https://oncouasd.files.wordpress.com/2014/09/ca ncer-principles-and-practice-of-oncology-6e.pdf

19. Rodríguez A, Gómez F, Álvarez J, Carballido J, Palou J, Solsona $\mathrm{E}$, et al. Factores que predicen el desarrollo de metástasis óseas por cáncer de próstata: recomendaciones de seguimiento y opciones terapéuticas. Actas Urol Esp. 2014; 38(4): 263-9. Doi: http://dx.doi.org/10.1016/j.acuro.2013.09.002

20. Ferrís J, García J, Berbel O, Ortega J. Factores de riesgo constitucionales en el cáncer de próstata. Actas Urol Esp. 2011; 35(5): 282-8. Doi: https://doi.org/10.1016/j.acuro.2010.12.009

21. Segarra J, Millán F, Palou J, Villavicencio H. Factores pronósticos y tablas predictivas del cáncer de próstata clínicamente localizado. Actas Urol Esp. 2006; 30(6): 567-73. Doi: https://doi.org/10.1016/S0210-4806(06)73496-9 
22. Pound C, Partin A, Epstein J, Walsh P. ProstateSpecific Antigen After Anatomic Radical Retropubic Prostatectomy: Patterns of Recurrence and Cancer Control. Urol Clin North Am. 1997 May; 24(2): 395-406. Doi: https://doi.org/10.1016/S00940143(05)70386-4

23. Oncology, American Society of Clinical. Cáncer de próstata: Estadísticas [Internet] [citado $11 \mathrm{abr}$ 2020]. Disponible en:

https://www.cancer.net/es/tipos-dec\%C3\%A1ncer/c\%C3\%A1ncer-depr\%C3\%B3stata/estad\%C3\%ADsticas 